Review

\title{
Therapeutic potential of mesenchymal stem/stromal cells-derived exosomes in acute respiratory distress syndrome and COVID-19
}

Authors:

Yueyuan Zhou ${ }^{1,2}$, Yusuke Yamamoto ${ }^{2}$, Zhongdang $\mathrm{Xiao}^{1^{*}}$, Takahiro Ochiya ${ }^{2,3^{*}}$

Affiliations:

1. State Key Laboratory of Bioelectronics, School of Biological Science and Medical Engineering, Southeast University, Nanjing 210096, China

2. Division of Cellular Signaling, National Cancer Center Research Institute, 51-1 Tsukiji, Chuo-ku, Tokyo 104-0045, Japan.

3. Department of Molecular and Cellular Medicine, Institute of Medical Science, Tokyo Medical University, 6-1-1 Shinjuku, Shinjuku-ku, Tokyo 1608402, Japan

\section{*Corresponding Author}

Zhongdang Xiao,

State Key Laboratory of Bioelectronics, School of Biological Science and Medical

Engineering, Southeast University

Tel: +86-025-83790820; E-mail: zdxiao@seu.edu.cn

Takahiro Ochiya,

Division of Molecular and Cellular Medicine, National Cancer Center Research

Institute

Department of Molecular and Cellular Medicine, Institute of Medical Science,

Tokyo Medical University

Tel: +81-3-3342-6111 (ext. 6168); Fax: +81-3-3543-9305; E-mail: tochiya@tokyomed.ac.jp

\begin{abstract}
Coronavirus disease 2019 (COVID-19) caused by novel severe acute respiratory syndrome coronavirus 2 (SARS-CoV-2) has reached a global epidemic across the world after first reported in Wuhan, China's Hubei province in December 2019. The pandemic is also associated with acute respiratory distress syndrome (ARDS) characterized by excess inflammation, progressive arterial hypoxemia and dyspnea. Mesenchymal stem/ stromal cells (MSCs) have been investigated as treatment for ARDS due to immunomodulatory property. Exosomes derived from MSCs play an important role in paracrine signaling of MSCs, thereby contributed to immunomodulation of the immune microenvironment. Exosomes are emerged as potential alternative to MSC cell therapy with superiority of safety. In this review, we will introduce MSC-derived exosomes and briefly discuss current progress on MSCs and exosomes in ARDS, which may have clinical implications in pathogenesis and treatment of COVID-19.
\end{abstract}

Keywords: Acute respiratory distress syndrome; Mesenchymal stem/stromal cells; exosomes; COVID-19; clinical trials 
2 of 18

\section{Introduction}

After the first report of infection in Wuhan in December 2019, Coronavirus disease 2019 (COVID-19) induced by severe acute respiratory syndrome coronavirus 2 (SARS-CoV-2) has spread to more than two hundred countries and claimed nearly 1.4 million lives throughout the world till November 23 $3^{\text {rd }}, 2020$ [1] [2] [3]. Mild clinical symptoms of COVID-19 including fever, dry cough, fatigue, headache, and pneumonia, whereas severe conditions result in dyspnea, ARDS, low level of oxygen in arterial blood, and multiple organ failure with a high-mortality rate [2] [4]. Currently, few available antiviral drugs show therapeutic effects for this disease. These characteristics of COVID-19 make it a challenge for treatment.

Mesenchymal stem/stromal cells (MSCs) are multipotent non-hematopoietic cells which can be isolated from both fetal and adult sources, such as the umbilical cord, adipose tissue, bone marrow, and dental pulp [5] [6]. In addition to capabilities of differentiating into various cell types, MSCs have broad immune regulatory property by interacting with immune system and influence both adaptive and innate immune responses [7] [8] [9]. Exosomes derived from MSCs echo the phenotype of their parental cells and contribute to the therapeutic effects of MSCs [10]. Hence, they are promising candidate approach for treatment of ARDS and severe COVID19. In this review, we will discuss therapeutic potential of exosomes derived from MSCs for ARDS and severe COVID-19.

ARDS induced by COVID-19

Since the first description in 1967, acute respiratory distress syndrome (ARDS) has been acknowledged as an acute form of diffuse lung injury resulting in increased alveolar capillary permeability, increased lung weight, and loss of aerated lung tissue [11] [12]. It is clinically characterized by hypoxemia with bilateral opacities on chest radiography, decreased lung compliance, increased venous admixture and physiological dead space [13]. Morphologically, diffuse alveolar damage is observed in the acute phase of ARDS. Severe COVID-19 represents viral pneumonia from SARS-CoV-2 infection leading to ARDS. After COVID-19 is confirmed by a consistent clinical history, epidemiological contact and positive SARS-CoV-2 test, COVID-19 ARDS is followingly diagnosed when it meets the Berlin 2012 ARDS diagnostic criteria of (i) acute hypoxaemic respiratory failure; (ii) presentation within 1 week of worsening respiratory symptoms; (iii) bilateral airspace disease on chest x-ray, computed tomography (CT) or ultrasound that is not fully explained by effusions, lobar or lung collapse, or nodules; and (iv) cardiac failure is not the primary cause of acute hypoxemic respiratory failure [13] [14]. Compared with the other serious coronavirus infections of severe acute respiratory syndrome and Middle East respiratory syndrome, COVID-19 displays an ongoing worldwide throat because the novel virus has the potential to mutate and infect non-immune populations. The typical ARDS pathological changes of diffuse alveolar damage in the lung is induced in COVID-19 ARDS [15] [16]. However, vascular enlargement is rarely seen in typical ARDS, while it is common in most cases of COVID-19 ARDS [17]. In most fatal cases of COVID-19 ARDS, there is evidence of thrombotic disseminated intravascular coagulation, indicating a thrombotic microangiopathy 
[18]. Besides, COVID-19 ARDS have worse clinical outcomes than ARDS resulted from other factors. Unadjusted ICU and hospital mortality from typical ARDS were $35.3 \%$ (95\% CI, 33.3\%-37.2\%) and 40\% (95\% CI, 38.1\%-42.1\%), respectively [19]. For COVID-19 ARDS, intensive care unit mortality ranged from $26.4 \%$ and $61.5 \%$, whereas mechanical ventilation mortality were between $65.7 \%$ and $94 \%$ [20]. Compared to non-COVID-19 ARDS, COVID-19 pneumonia was more likely to have a peripheral distribution ( $80 \%$ vs. $57 \%$, p $<0.001)$, ground-class opacity $(91 \%$ vs. $68 \%$, $\mathrm{p}<0.001)$, fine reticular opacity ( $56 \%$ vs. $22 \%, \mathrm{p}<0.001)$, and vascular thickening $(59 \%$ vs. $22 \%$, p $<0.001)$, but less likely to have a central + peripheral distribution (14\% vs. $35 \%, \mathrm{P}<0.001)$, pleural effusion $(4.1 \%$ vs. $39 \%, \mathrm{p}<0.001)$ and lymphadenopathy $(2.7 \%$ vs. $10.2 \%, \mathrm{p}<0.001$ ) [21]. These discriminating features from chest radiograph tend to help radiologists distinguish COVID-19 from viral pneumonia with high specificity. The ventilation strategy is critical in treatment of COVID-19 ARDS as well as typical ARDS cases resulted from other pathogens [14]. Non-invasive ventilation helped offer positive end-expiratory pressure (PEEP), reduce respiratory load and intubation rate in treating mild and moderate ARDS, as is in COVID-19 induced moderate ARDS [22] [23]. However, it is required that close observation and flexible adjustment in non-invasive ventilation. Lack of medical staff and rapid increase of patient number make it hard to operate in COVID-19 ARDS. Prone ventilation and extracorporeal membrane oxygen (ECMO) did not appear to be effective in COVID-19 ARDS patients in Wuhan [24]. Possible associated factors include lung low retensibility resulted from alveolar lesions, endogenous lung lesions and abnormal immune status induced by COVID-19.

Properties of MSC-derived exosomes

Due to their capacity to regulate phenotype and function of immune cells, mesenchymal stem/stromal cells (MSCs) have been emerged as promising candidate for the treatment of autoimmune and inflammatory diseases [25]. There is mounting evidence that MSC secretome, including soluble components, and encapsulated extracellular vesicles mainly contributed to immunoregulatory properties of MSCs [26] [27] [28]. Recent studies revealed that both local and systemic administration of MSC- exosomes efficiently suppressed detrimental immune response in inflamed tissues. MSC- exosomes-based immunoregulatory effects were dependent on the delivery of immunomodulatory miRNAs and proteins in inflammatory immune cells (M1 macrophages, dendritic cells (DCs), CD4+ Th1 and Th17 cells), inducing their phenotypic conversion into immunosuppressive M2 macrophages, tolerogenic DCs and T regulatory cells [29] [30] [31]. Exosomes derived from MSCs shared the common characters with exosomes derived from other cell types. Typically, exosomes have a diameter of $40-100 \mathrm{~nm}$ with a density of $1.13-1.19 \mathrm{~g} / \mathrm{mL}$ in a sucrose solution [32]. 

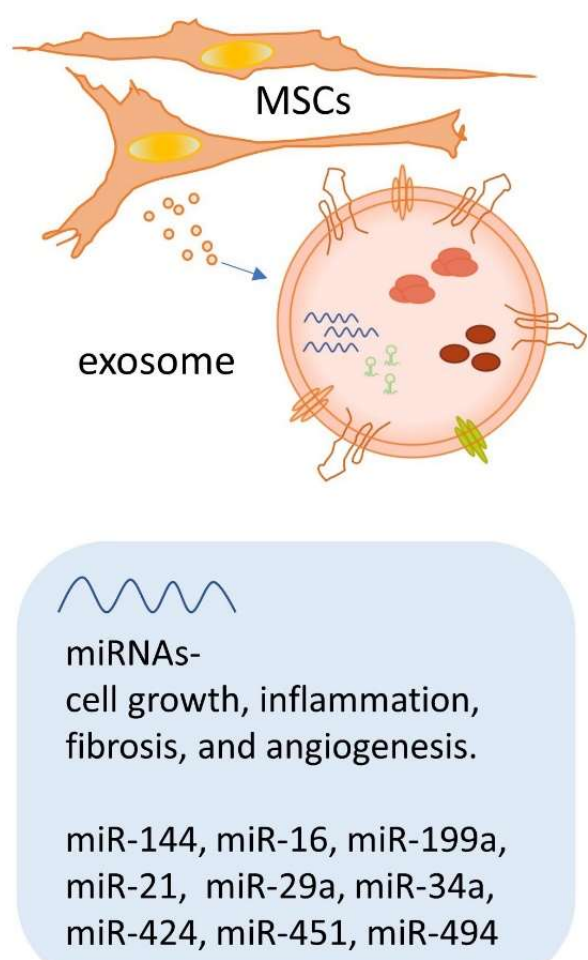

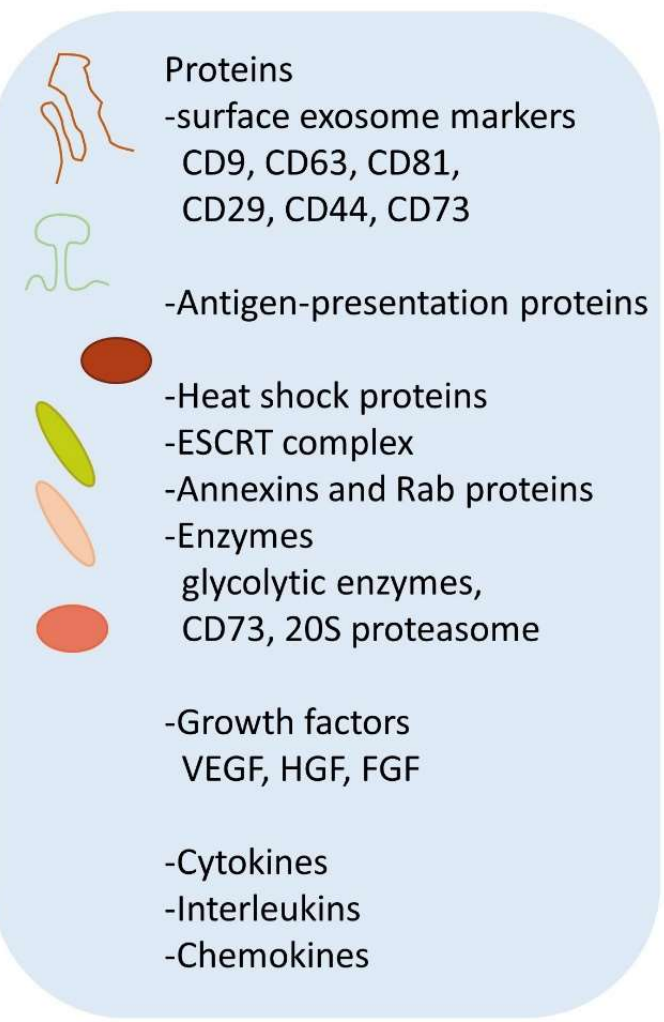

\section{Figure 1. Characteristics of MSC-derived exosomes}

MSC-exosomes express some adhesion molecules, such as CD29. CD44 and CD73, which are expressed on the membrane of MSCs in addition to common surface markers of exosomes, including CD9, CD63 and CD81 [33] [34]. Heat shock proteins and proteins related to exosome biogenesis such as ESCRT complex, proteins involved in transcription, and protein synthesis such as ribosomal protein, and membrane fusion proteins like Annexins were founded in MSC-exosomes. In addition, other proteins capsulated in MSC-exosomes include luminal proteins like annexin2, antigen-presentation proteins like MHC-I and MHC-II, cell adhesion molecules like integrin and MFGE8, co-presentation proteins CD86, and cell structure and motility proteins including actin, myosin and tubulin [35]. Proteomic studies have shown that MSC-exosomes contain enzymes involved in glycolysis such as enolase, pyruvate kinase $\mathrm{m} 2$ isoform (PKm2) and other enzymes such as ecto-59-nucleotidase (or NT5E or CD73) and 20S proteasome [36] [37]. The presence

of $20 \mathrm{~S}$ proteasome provides a potential molecular mechanism for the cardioprotective activity of MSC-exosomes by mass spectrometric analysis [38]. It was reported that MSC-exosomes exerted therapeutic effects in myocardial infarction through growth factors including VEGF, HGF, and FGF and cytokines as colony-stimulating factors [39] [40]. The protein components are not constant in exosomes that are isolated from MSC conditioned media which are collected from different batches. It was reported that there are 379,432 , and 420 proteins detected 
in MSC-exosomes isolated from three independent batches by liquid chromatography-mass spectrometry, and only 154 proteins are common [38]. MSCexosomes express several adhesion molecules, which enable them to home to the injured and inflamed tissues. It was found that MSC-exosomes mainly accumulated in the inflamed kidneys in the acute kidney injury mouse model [41]. The membrane fusion process of exosome with target cells is relied on cholesterol, sphingomyelin, ceramide and lipid raft proteins which are enriched in the outer membrane of exosomes, making exosomes free from biological barriers [42]. MSC-exosomes could cross the blood-brain barrier (BBB) since they were detected in the brains following injection through the tail vein in the rat model of intracerebral hemorrhage, and promoted brain functional recovery [43]. Most of intravenously injected MSCexosomes accumulated in the liver, spleen and lung [43]. Hence, several researches focused on membrane-editing technology to modify surface of MSC-exosomes in order to realize specific targeting before exosomes were captured by macrophages [44] [45].

MicroRNAs are another focus which has attracted much attention in the studies of MSC-exosomes. It has been proved that miRNAs contained in MSC-exosomes are mainly in their precursor form [46]. MSC-exosomes mediate cell-to-cell communication partly through transferring miRNAs. Microarray assessment of exosome RNA showed that around 1300 different RNAs re encapsulated in exosomes [47]. MSC-exosomes helped recover renal function in an aminoglycoside acute kidney injury (AKI) model, which could be inhibited by RNase [48]. MiR-451 in MSC-exosomes negatively regulated cytokine production in macrophages and contributed to attenuation of monocyte activation [49]. It was investigated that MSCexosomes promoted myogenesis and angiogenesis in vitro, and muscle regeneration in vivo partly through delivering miR-494 [50]. It was also indicated that MSCexosomes could repair cisplatin-induced AKI by ameliorating oxidative stress and cell apoptosis, and promoting cell proliferation [51]. In our previous work, we analyzed common miRNAs in exosomes derived from human bone marrow, adipose tissue, Wharton's jelly and exfoliated deciduous teeth [52]. In the listed 8 common miRNAs that are biologically well-documented, miR-199a-3p downregulation showed protective effects in sepsis-induced ARDS by upregulation of SIRT1 through the suppression of excessive inflammatory responses and inhibition of cellular apoptosis in lung tissue [53]. However, Chen et al. reported that miR-199a-3p downregulation promoted the secretion of proinflammatory IL-1 $\beta$ and IL-18 by targeting NLR1, leading to acute lung injury pathogenesis [54]. These findings reveal that miR-199a-3p in MSC-exosomes may have pro- and antiinflammatory effects in different disease models. It was reported that miR-29a activated DCs and improved liver inflammation and fibrosis [55] [56]. MiR-23a-3p in serum was significantly lower in patients with sepsis-induced AKI and was correlated with mitochondrial oxidative stress and dysfunction [57]. Exosomes derived from different tissue source MSCs expressed distinct miRNAs which are involved in differential differentiation [52]. MiR-144-3p specifically expressed in exosomes derived from Wharton's jelly MSCs was found to increase in LPS-induced 
ALI mice [58] and promoted fibrosis through relaxin/insulin-like family peptide receptor 1 (RXFP1) in lung fibroblasts [59]. The miRNA profiles of MSC-exosomes may predict their targeted cell groups and possible functions.

Mechanisms of therapeutic benefits of MSC-derived exosomes in ARDS

MSCs are multipotent progenitor cells with anti-inflammatory and immunomodulatory capabilities which exist in nearly all forms of post-natal organs and tissues [60]. Mesenchymal epithelial interactions have a critical role in lungs. MSCs derived from lung were crucial for epithelial differentiation and morphogenesis [61]. And co-culture of embryonic stem cell with lung mesenchyme promoted their differentiation to alveolar epithelium [62]. The bidirectional regulation of the epithelial and stromal components by soluble cytokines was well documented [63] [64] [65]. It was investigated that MSCs derived from human bronchoalveolar lavage fluid of lung transplant recipients persisted in murine lung for 6 months and preferentially localized to alveolar epithelial cells [66]. The in-vitro evidence revealed the gap junction communications between MSCs, lung alveolar and bronchial epithelial cells. And MSCs in the alveolar microenvironment promoted epithelial cell proliferation and differentiation by secreting keratinocyte growth factor (KGF) [66]. Paracrine signaling of MSCs not only contributed to their regulation on alveolar epithelial cells, but also stimulating lung fibroblast growth [67].

Recent experimental studies have shown that intratracheal or intravenous administration of MSC-exosomes could attenuate acute lung injury [68] [69]. Through transferring miRNAs, MSC-exosomes reduced secretion of inflammatory cytokines and monocyte infiltration into the lung [49]. MSC-exosome-based protection of lung epithelial cells against oxidative stress-induced cell death was dependent on anti-apoptotic properties of miR-21-5p [70]. In addition to antioxidative effects, protease/ antiprotease balance regulated by exosomes was involved in the lung protection. Adipose-derived-MSC-exosomes contained alpha1-antitrypsin (AAT), the main elastase inhibitor in the lung and 72 other proteins related to protease/ antiprotease balance [71]. Besides, another 46 proteins involved in the response to Gram-negative bacteria were delivered by MSC-exosomes, suggesting potent anti-microbial capability of MSC-exosomes [71]. Findings consistent with these results were reported that administration of MSC-exosomes significantly reduced severity of bacterial pneumonia in mice with acute lung injury [72]. MSC-exosomes suppressed expression of multidrug resistance-associated protein 1 (MMP1) mRNA and protein through transfer of miR-145, induced production of leukotriene (LT) $\mathrm{B}_{4}$, thereby enhanced phagocytic and anti-microbial activity of lung-infiltrating neutrophils and monocytes [72]. Following the phase of anti-microbial inflammatory response, MSC-exosomes helped modulate phenotype and function of alveolar macrophages. During the resolution of inflammation, MSCexosomes downregulated iNOS mRNA expression and upregulated Arginase-1 mRNA in alveolar macrophages, inducing their polarization from inflammatory M1 towards immunosuppressive M2 phenotype [73]. Therefore, secretion of M1-related inflammatory cytokines (IL-8, IL-1 $\beta$, IL-6 and TNF- $\alpha$ ) was decreased, whereas M2 
macrophages-released immunosuppressive cytokines (IL-10 and TGF- $\beta$ ) were increased in murine lung ischemia/ reperfusion model [70]. Similarly, treatment with MSC-exosomes significantly decreased the proinflammatory cytokines IL-17, TNF- $\alpha$, CXCL1 and HMGB1 and increased KGF, prostaglandin E2 (PGE2), IL-10 in the bronchoalveolar lavage fluid from mice [74]. In addition to alveolar macrophages, MSC-exosomes may also modulate phenotype and function of lunginfiltrating dendritic cells (DCs). MSC-exosomes mediated increased production of immunosuppressive IL-10 and TGF- $\beta$ suppressed maturation of lung DCs, and immature DCs reduced expression of co-stimulatory molecules (CD40, CD80 and CD86), leading to inactivation of CD4+ Th2 cells and alleviation of Th2-driven lung inflammation [75].

Taken together, MSC-exosomes exerted anti-inflammatory therapeutic effects via modulation of phenotype and function of antigen-presenting cells, attenuation of immune cell activation as well as prevention of endothelial barrier integrity to prevent lung edema.
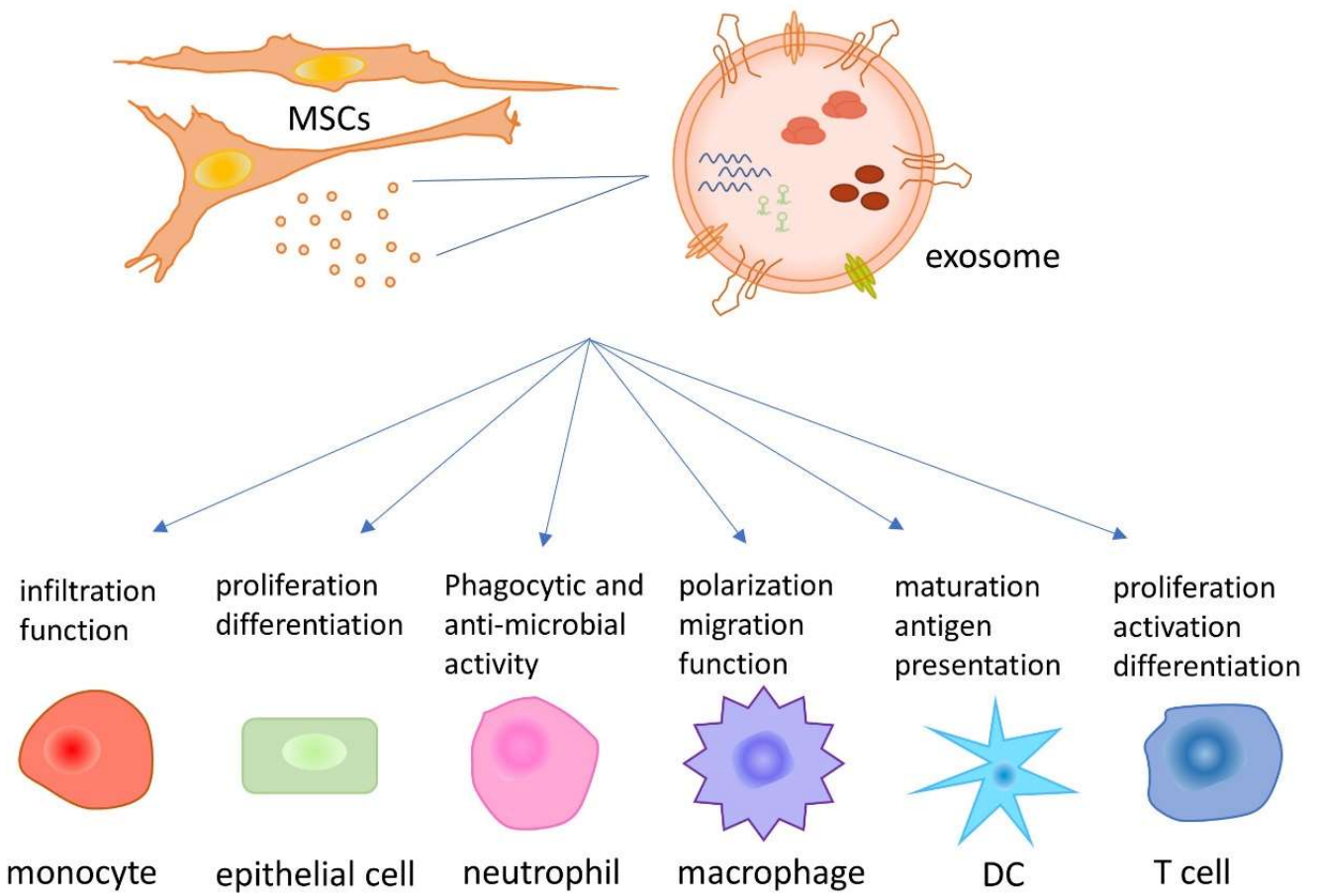

Figure 2. Graphic summary of effects of MSC-derived exosomes in ARDS and COVID-19. MSCs and their exosomes have a potent ability to modulate monocytes, lung epithelial cells and immune cells.

Clinical Trials of MSC-derived exosomes in Patients with ARDS and COVID-19

Recently, few reports have demonstrated MSCs and exosomes in treatment of ARDS and COVID-19. One of the earliest studies to examine the safety of MSCs in treatment of ARDS reported that there were no infusion toxicities or serious adverse 
events related to MSCs administration. Compared to placebo group, MSC-treated group showed significantly lower serum surfactant protein D on $5^{\text {th }}$ day than on day 0 . However, the clinical effects of using MSC does were weak, and this strategy required to be optimized (NCT01902082) [76]. In the single-center, randomized Russian clinical trial of MSCs (NCT01849237) in severe neutropenic patients with septic shock, patients were randomly assigned to receive conventional therapy (CT) or CT plus donor MSCs at a dose of $1 \times 10^{6} / \mathrm{kg}$ intravenously within $10 \mathrm{~h}$ after septic shock onset. All patients except for one with myelodysplastic syndrome developed neutropenia after chemotherapy. Most of the positive blood cultures from patients were gram-negative. Despite higher 28-day survival rates only 3 patients treated with CT plus MSCs remained alive after 3 months, 5 of 8 patients who survived 28 days died later because of sepsis-related organ dysfunction. These results indicated that MSC administration in the first hours of septic shock might improve short-term survival in neutropenic patients, but did not prevent death from sepsis-related organ dysfunction in the long term [77]. In a multicenter, open-label, dose-escalation, phase 1 clinical trial (NCT01775774), nine patients with moderate-to-severe ARDS tolerated a single intravenous infusion of human MSCs derived from allogeneic bone marrow without prespecified infusion-associated events or treated-related adverse events. Serious adverse events were subsequently noted in three patients during the weeks after the infusion but were thought to occur before MSC injection. Further phase 2 testing were required to investigate the safety and secondary outcomes including respiratory, systemic, and biological endpoints [78]. Simonson et al. performed a detailed analysis of the immunomodulatory properties and proteomic profile of MSCs systemically administered to two patients with severe refractory ARDS. Both patients received $2 \times 10^{6}$ cells $/ \mathrm{kg}$, and subsequently showed improved with resolution of respiratory, hemodynamic, and multiorgan failure. The inflammation was attenuated with declined lung and systemic inflammatory markers including epithelial cell apoptosis, leakage of alveolar-capillary fluid, proinflammatory cytokines, miRNAs and chemokines. These findings suggested a beneficial effect of lung protective strategies using adoptively transferred MSCs in ARDS [79]. Another multicenter, randomized phase 2a trial (NCT02097641) proved that for patients with moderate-to-severe ARDS, a single intravenous dose of MSCs is safe [80]. Based on this result, it was further demonstrated that the therapeutic effects of MSC treatment were depending on the patient's specific pulmonary microenvironment, including the levels of IL-6 and fibronectin and total antioxidant capacity (TAC). MSC administration into mice was protective in settings with low concentrations of IL-6 and fibronectin and high level of TAC. This conceptional framework provided a strong rationale for a precision medicine approach for MSC treatment [81]. We also searched ongoing related clinical studies on ClinicalTrials.gov. and identified 4 completed and 31 recruiting trials (Supplementary table: Table 1). 17 trials (48.6\%) used MSCs derived from umbilical cord tissue, 6 trials (17.1\%) used MSCs from bone marrow, 2 trials (5.7\%) used adipose tissue-derived MSCs, and 1 trial (2.9\%) used dental pulp-derived MSCs. Almost all trials are phase I, II, or I/II studies (One trial is phase II/III, and one is 
phase III). A pilot clinical study registered in China was performed to explore the safety and efficiency of aerosol inhalation of exosomes derived from allogenic adipose tissue MSCs in severe patients with COVID-19 (NCT04276987). Another prospective nonrandomized open-label cohort study demonstrated ExoFlo, a bone marrow-derived MSC exosome agent, could be administered safely through intravenous infusion. The study met all of its primary endpoints. All patients were administered ExoFlo without any infusion reaction and adverse effects [82]. There are crucial weaknesses in current reporting standards for primary clinical outcomes. Deaths in ill patients may be attributed to many possible causes and may not be preventable with standard operating procedures. The beneficial effects of proposed treatments and the long-term outcome, including survival and quality of life remain incredibly hard to detect and assess. Further follow-up studies on survivors of ARDS reveal long-term neuromuscular and psychiatric disorders that developed in patients with an assumed complete initial recovery from ARDS [83].

Compared to MSCs, exosomes would be inherently safer for intravenous administration to patients and the risk of tumor formation would be much lower. Additionally, exosomes would be less immunogenic as they do not carry MHC I and II class antigens. Modification and enrichment of a particular subset of exosomes by pretreatment of MSC source could also improve their potency. Overexpression of miR-30b-3p in MSC-exosomes conferred protective effects against acute lung injury (ALI) by downregulating serum amyloid A3 expressed in alveolar epithelial cells [84]. However, since these modifications may lead to undesirable changes in exosomes, the effects and safety of modified exosomes should be assessed independently. Exosomes can keep stable and be cryopreserved for a long time without alteration of biological activity. Besides, the small size provides exosomes the advantage of protection from microcirculation obstruction. Coated with phospholipid membrane, exosomes carry a variety of bioactive components which cannot be easily degraded by enzymes.

Unsolved frontiers

MSCs and MSC-exosomes are attractive potential therapy of diverse diseases including ARDS with COVID-19. Preliminary animal and clinical studies report promising findings; however, they have severe methodological limitations and heterogeneity. There are several unsolved questions: the donor, cell source of MSCs; the fresh or cryopreserved and original or expanded cells; the dose, route and frequency; the isolation and characterization of exosomes. A phase 1/2 randomized comparison study addressed the issue of allogeneic vs. autologous MSC therapy for ischemic cardiomyopathy (NCT01087996) [85]. Allogeneic MSCs did not stimulate significant donor-specific alloimmune reactions. However, autologous but not allogeneic MSCs were associated with an improvement in the 6-minute walk test and the Minnesota Living with Heart Failure Questionnaire (MLHFQ) score. Lowdose concentration MSCs $\left(2 \times 10^{6}\right)$ produced greatest reductions in left ventricular (LF) volumes and increased ejection fraction (EF). It was reported that transplanted MSCs from healthy donors with no known history of autoimmune disease are immunosuppressive in systemic lupus erythematosus (SLE) patients and can 
ameliorate SLE disease symptoms in those same patients. In contrast, autologous MSCs from SLE patients are not immunosuppressive and do not ameliorate disease symptoms [86]. These results suggested that further prospective studies are required to evaluate autogenous vs. allogeneic source of MSCs as the exosome cell source for treatment of ARDS with COVID-19. MSCs were originally isolated from bone marrow, and they have also been found in other tissues such as adipose tissue, umbilical cord, cord blood, skeletal muscle and lung [87]. Homogenous MSCs from different tissues presented different differentiation trend and phenotype and function. HEO et al. compared the immunotype, proliferative potential, multilineage differentiation and immunomodulatory capacity of MSCs derived from bone marrow, adipose tissue, the placenta and umbilical cord blood [88]. They investigated expression files of genes related to stemness, lineage, differentiation stage and compared growth rate, colony-formation and immunophenotype. Their results demonstrated that MSCs derived from bone marrow significantly inhibited allogeneic $\mathrm{T}$ cell proliferation possibly via immunosuppressive cytokines IL-10 and TGF- $\beta 1$, suggesting MSCs from bone marrow and adipose tissue represent the optimal stem cell source for tissue engineering and regenerative medicine. In addition, it was reported that MSCs from umbilical cord produced the highest exosome yield compared to MSCs from bone marrow and adipose tissue [89]. And in combination with the conventional differential ultracentrifugation, threedimensional (3D) culture based on scalable microcarrier yields 20-fold more exosomes. Similarly, Cao et al. investigated a strategy to increase total MSC-exosome production to 19.4-fold with a hollow fiber bioreactor-based 3D culture system and evaluated the therapeutic efficacy of 3D-exosomes om AKI [90]. They reported that 3D culture did not significantly change the surface markers of MSCs, as well as the morphology, size, and exosomal markers. 3D-exosomes displayed more effective roles evidenced by improved renal function, attenuated pathological changes of renal tubules, reduced inflammatory factors, and repressed $\mathrm{T}$ cell and macrophage infiltration. Intravenous systematic delivery of MSC and exosomes has been the most common route evaluated in clinical trials since it is an acceptable and logistically feasible way for a COVID-19 infection. It has been revealed that treatment of exosomes from lung spheroid cells by inhalation could attenuate bleomycin-and silica-induced fibrosis, and decrease both collagen accumulation and myofibroblast proliferation, hence promote lung repair in pulmonary fibrosis [91]. There is no randomized data available to evaluate the optimal route of delivery in human trials. The heterogeneous subpopulations of exosomes make a challenge to comply with good manufacturing practice (GMP). It is critical to focus on three main criteria, including upstream of cell cultivation system, downstream of the purification system and quality control of exosomes. The GMP-grade exosome production methods are generally divided into several facets: cell type, culture environment, cultivation system, dissociation enzyme and culture medium [92]. Further purification is required after production. The third issue is establishment of identification method to characterize physical structure and bioactivity function. The limited timelines to treat accelerating case numbers strengthen the hardness in 
MSC-exosomes therapy for COVID-19. Therefore, it is urgent to apply further studies on a safe off-the-shelf product with appropriate immune response modulation activity, which allows patients to receive the best quality cell-free treatment.

\section{Conclusion and Perspectives}

MSC-exosomes are increasingly potential candidate for the treatment of ARDS and COVID-19. Preclinical studies provided favorable therapeutic benefits of MSCexosome treatment. Considering their cell source MSCs, unanswered questions are listed: the donor and source; the dose, route and frequency; fresh cells or cryopreserved cells; primary cells or expanded passages. As for exosomes themselves, their poorly understood bioactive contents, function and associated molecular mechanisms with which they communicate with inflammatory immune cells and stromal cells in the damaged lung microenvironment. Large-scale production of exosomes requires a large number of MSC cells and massive culture medium, which is costly. Further investigations into these facets of the biology of MSC-exosomes will help build our preparations for the prolonged COVID-19 pandemic.

\section{Authors' contributions}

Yueyuan Zhou searched for information and wrote the paper. Yusuke Yamamoto, Takahiro Ochiya, and Zhongdang Xiao reviewed and edited the manuscript. All authors read and approved the manuscript.

\section{Conflicts of Interest}

The authors declare that they have no competing interests.

\section{References}

1. Huang, C.; Wang, Y.; Li, X.; Ren, L.; Zhao, J.; Hu, Y.; Zhang, L.; Fan, G.; Xu, J.; Gu, $\mathrm{X}$; et al. Clinical features of patients infected with 2019 novel coronavirus in Wuhan, China. Lancet 2020, 395, 497-506, doi:10.1016/S0140-6736(20)30183-5.

2. Chen, N.; Zhou, M.; Dong, X.; Qu, J.; Gong, F.; Han, Y.; Qiu, Y.; Wang, J.; Liu, Y.; Wei, Y.; et al. Epidemiological and clinical characteristics of 99 cases of 2019 novel coronavirus pneumonia in Wuhan, China: a descriptive study. Lancet 2020, 395, 507513, doi:10.1016/S0140-6736(20)30211-7.

3. Coronavirus Disease (COVID-19) Pandemic.

4. Wong, J.; Abdul Aziz, A.B.Z.; Chaw, L.; Mahamud, A.; Griffith, M.M.; Lo, Y.-R.; Naing, L. High proportion of asymptomatic and presymptomatic COVID-19 infections in air passengers to Brunei. Journal of Travel Medicine 2020, 27, doi: $10.1093 / \mathrm{jtm} / \mathrm{taaa} 066$.

5. PS in 't Anker; WA Noort; SA Scherjon; C Kleijburg-van der Keur; AB Kruisselbrink; RL van Bezooijen; W Beekhuizen; R Willemze; HH Kanhai; WE Fibbe Mesenchymal stem cells in human second-trimester bone marrow, liver, lung, and spleen exhibit a similar immunophenotype but a heterogeneous multilineage differentiation potential. haematol 2003, 88, 845-852, doi:10.3324/\%x.

6. Ponnaiyan, D.; Bhat, K.M.; Bhat, G.S. Comparison of Immuno-Phenotypes of Stem Cells from Human Dental Pulp and Periodontal Ligament. Int $J$ Immunopathol Pharmacol 2012, 25, 127-134, doi:10.1177/039463201202500115.

7. Le Blanc, K.; Mougiakakos, D. Multipotent mesenchymal stromal cells and the innate immune system. Nature Reviews Immunology 2012, 12, 383-396, doi:10.1038/nri3209. 
8. Bernardo, M.E.; Fibbe, W.E. Mesenchymal Stromal Cells: Sensors and Switchers of Inflammation. Cell Stem Cell 2013, 13, 392-402, doi:10.1016/j.stem.2013.09.006.

9. Li, N.; Hua, J. Interactions between mesenchymal stem cells and the immune system. Cellular and Molecular Life Sciences 2017, 74, 2345-2360, doi:10.1007/s00018-0172473-5.

10. Katsuda, T.; Kosaka, N.; Takeshita, F.; Ochiya, T. The therapeutic potential of mesenchymal stem cell-derived extracellular vesicles. PROTEOMICS 2013, 13, 16371653, doi:10.1002/pmic.201200373.

11. Ashbaugh, DavidG.; Boyd Bigelow, D.; Petty, ThomasL.; Levine, BernardE. Acute respiratory distress in adults. The Lancet 1967, 290, 319-323, doi:10.1016/S01406736(67)90168-7.

12. Rubenfeld, G.D.; Caldwell, E.; Peabody, E.; Weaver, J.; Martin, D.P.; Neff, M.; Stern, E.J.; Hudson, L.D. Incidence and Outcomes of Acute Lung Injury. N Engl J Med 2005, 353, 1685-1693, doi:10.1056/NEJMoa050333.

13. The ARDS Definition Task Force* Acute Respiratory Distress Syndrome: The Berlin Definition. JAMA 2012, 307, 2526-2533, doi:10.1001/jama.2012.5669.

14. Gibson, P.G.; Qin, L.; Puah, S.H. COVID-19 acute respiratory distress syndrome (ARDS): clinical features and differences from typical pre-COVID-19 ARDS. Med $J$ Aust 2020, 213, 54-56.e1, doi:10.5694/mja2.50674.

15. Xu, Z.; Shi, L.; Wang, Y.; Zhang, J.; Huang, L.; Zhang, C.; Liu, S.; Zhao, P.; Liu, H.; Zhu, L.; et al. Pathological findings of COVID-19 associated with acute respiratory distress syndrome. Lancet Respir Med 2020, 8, 420-422, doi:10.1016/S22132600(20)30076-X.

16. Tian, S.; Xiong, Y.; Liu, H.; Niu, L.; Guo, J.; Liao, M.; Xiao, S.-Y. Pathological study of the 2019 novel coronavirus disease (COVID-19) through postmortem core biopsies. Mod Pathol 2020, 33, 1007-1014, doi:10.1038/s41379-020-0536-x.

17. Achour, A.; Dkhil, O.; Saad, J.; Abdelali, M.; Zrig, A.; Hmida, B.; Golli, M.; Maatouk, M.; Mnari, W. Chest CT-scan finding of asymptomatic COVID-19 pneumonia: a prospective 542 patients' single center study. Pan Afr Med J 2020, 36, 257-257, doi:10.11604/pamj.2020.36.257.23632.

18. Ye, Z.; Zhang, Y.; Wang, Y.; Huang, Z.; Song, B. Chest CT manifestations of new coronavirus disease 2019 (COVID-19): a pictorial review. Eur Radiol 2020, 30, 4381 4389, doi:10.1007/s00330-020-06801-0.

19. Bellani, G.; Laffey, J.G.; Pham, T.; Fan, E.; Brochard, L.; Esteban, A.; Gattinoni, L.; van Haren, F.; Larsson, A.; McAuley, D.F.; et al. Epidemiology, Patterns of Care, and Mortality for Patients With Acute Respiratory Distress Syndrome in Intensive Care Units in 50 Countries. JAMA 2016, 315, 788-800, doi:10.1001/jama.2016.0291.

20. Wu, C.; Chen, X.; Cai, Y.; Xia, J.; Zhou, X.; Xu, S.; Huang, H.; Zhang, L.; Zhou, X.; Du, C.; et al. Risk Factors Associated With Acute Respiratory Distress Syndrome and Death in Patients With Coronavirus Disease 2019 Pneumonia in Wuhan, China. JAMA Intern Med 2020, 180, 934-943, doi:10.1001/jamainternmed.2020.0994.

21. Bai, H.X.; Hsieh, B.; Xiong, Z.; Halsey, K.; Choi, J.W.; Tran, T.M.L.; Pan, I.; Shi, L.B.; Wang, D.-C.; Mei, J.; et al. Performance of Radiologists in Differentiating COVID19 from Non-COVID-19 Viral Pneumonia at Chest CT. Radiology 2020, 296, E46E54, doi:10.1148/radiol.2020200823.

22. Agarwal, R.; Aggarwal, A.N.; Gupta, D. Role of Noninvasive Ventilation in Acute Lung Injury/Acute Respiratory Distress Syndrome: A Proportion Meta-analysis. Respiratory Care 2010, 55, 1653.

23. Nava, S.; Schreiber, A.; Domenighetti, G. Noninvasive Ventilation for Patients With Acute Lung Injury or Acute Respiratory Distress Syndrome. Respiratory Care 2011, 56, 1583, doi:10.4187/respcare.01209. 
24. Pan, C.; Xie, J.; Qiu, H. Therapy of coronavirus disease 2019 induced acute respiratory distress syndrome is different from traditional acute respiratory distress syndrome. Zhonghua Shao Shang Za Zhi 2020, 36, 330-333, doi:10.3760/cma.j.cn50112020200407-00214.

25. Regmi, S.; Pathak, S.; Kim, J.O.; Yong, C.S.; Jeong, J.-H. Mesenchymal stem cell therapy for the treatment of inflammatory diseases: Challenges, opportunities, and future perspectives. European Journal of Cell Biology 2019, 98, 151041, doi:10.1016/j.ejcb.2019.04.002.

26. Harrell, C.R.; Jankovic, M.G.; Fellabaum, C.; Volarevic, A.; Djonov, V.; Arsenijevic, A.; Volarevic, V. Molecular Mechanisms Responsible for Anti-inflammatory and Immunosuppressive Effects of Mesenchymal Stem Cell-Derived Factors. In Proceedings of the Tissue Engineering and Regenerative Medicine; Pham, P.V., Ed.; Springer International Publishing: Cham, 2019; pp. 187-206.

27. Zhou, Y.; Yamamoto, Y.; Xiao, Z.; Ochiya, T. The Immunomodulatory Functions of Mesenchymal Stromal/Stem Cells Mediated via Paracrine Activity. J Clin Med 2019, 8, 1025, doi: 10.3390/jcm8071025.

28. Emukah, C.; Dittmar, E.; Naqvi, R.; Martinez, J.; Corral, A.; Moreira, A.; Moreira, A. Mesenchymal stromal cell conditioned media for lung disease: a systematic review and meta-analysis of preclinical studies. Respir Res 2019, 20, 239-239, doi:10.1186/s12931-019-1212-x.

29. Biswas, S.; Mandal, G.; Roy Chowdhury, S.; Purohit, S.; Payne, K.K.; Anadon, C.; Gupta, A.; Swanson, P.; Yu, X.; Conejo-Garcia, J.R.; et al. Exosomes Produced by Mesenchymal Stem Cells Drive Differentiation of Myeloid Cells into Immunosuppressive M2-Polarized Macrophages in Breast Cancer. J Immunol 2019, 203, 3447-3460, doi:10.4049/jimmunol.1900692.

30. Shahir, M.; Mahmoud Hashemi, S.; Asadirad, A.; Varahram, M.; Kazempour-Dizaji, M.; Folkerts, G.; Garssen, J.; Adcock, I.; Mortaz, E. Effect of mesenchymal stem cellderived exosomes on the induction of mouse tolerogenic dendritic cells. $J$ Cell Physiol 2020, 235, 7043-7055, doi:10.1002/jcp.29601.

31. Chen, W.; Huang, Y.; Han, J.; Yu, L.; Li, Y.; Lu, Z.; Li, H.; Liu, Z.; Shi, C.; Duan, F.; et al. Immunomodulatory effects of mesenchymal stromal cells-derived exosome. Immunologic Research 2016, 64, 831-840, doi:10.1007/s12026-016-8798-6.

32. Breakefield, X.O.; Frederickson, R.M.; Simpson, R.J. Gesicles: Microvesicle "cookies" for transient information transfer between cells. Mol Ther 2011, 19, 1574-1576, doi:10.1038/mt.2011.169.

33. Chaput, N.; Théry, C. Exosomes: immune properties and potential clinical implementations. Seminars in Immunopathology 2011, 33, 419-440, doi:10.1007/s00281-010-0233-9.

34. Mohammadipoor, A.; Antebi, B.; Batchinsky, A.I.; Cancio, L.C. Therapeutic potential of products derived from mesenchymal stem/stromal cells in pulmonary disease. Respir Res 2018, 19, 218-218, doi:10.1186/s12931-018-0921-x.

35. Deng, H.; Sun, C.; Sun, Y.; Li, H.; Yang, L.; Wu, D.; Gao, Q.; Jiang, X. Lipid, Protein, and MicroRNA Composition Within Mesenchymal Stem Cell-Derived Exosomes. Cellular Reprogramming 2018, 20, 178-186, doi:10.1089/cell.2017.0047.

36. Steelman, L.S.; Chappell, W.H.; Abrams, S.L.; Kempf, C.R.; Long, J.; Laidler, P.; Mijatovic, S.; Maksimovic-Ivanic, D.; Stivala, F.; Mazzarino, M.C.; et al. Roles of the Raf/MEK/ERK and PI3K/PTEN/Akt/mTOR pathways in controlling growth and sensitivity to therapy-implications for cancer and aging. Aging (Albany NY) 2011, 3, 192-222, doi:10.18632/aging.100296. 
37. Lai, R.C.; Chen, T.S.; Lim, S.K. Mesenchymal stem cell exosome: a novel stem cellbased therapy for cardiovascular disease. Regenerative Medicine 2011, 6, 481-492, doi:10.2217/rme.11.35.

38. Lai, R.C.; Tan, S.S.; Teh, B.J.; Sze, S.K.; Arslan, F.; de Kleijn, D.P.; Choo, A.; Lim, S.K. Proteolytic Potential of the MSC Exosome Proteome: Implications for an Exosome-Mediated Delivery of Therapeutic Proteasome. Int J Proteomics 2012, 2012, 971907-971907, doi:10.1155/2012/971907.

39. Zgheib, C.; Zouein, F.A.; Kurdi, M.; Booz, G.W. Chronic treatment of mice with leukemia inhibitory factor does not cause adverse cardiac remodeling but improves heart function. Eur Cytokine Netw 2012, 23, 191-197, doi:10.1684/ecn.2012.0319.

40. Formiga, F.R.; Pelacho, B.; Garbayo, E.; Imbuluzqueta, I.; Díaz-Herráez, P.; Abizanda, G.; Gavira, J.J.; Simón-Yarza, T.; Albiasu, E.; Tamayo, E.; et al. Controlled delivery of fibroblast growth factor-1 and neuregulin-1 from biodegradable microparticles promotes cardiac repair in a rat myocardial infarction model through activation of endogenous regeneration. Journal of Controlled Release 2014, 173, 132-139, doi:10.1016/j.jconrel.2013.10.034.

41. Grange, C.; Tapparo, M.; Bruno, S.; Chatterjee, D.; Quesenberry, P.J.; Tetta, C.; Camussi, G. Biodistribution of mesenchymal stem cell-derived extracellular vesicles in a model of acute kidney injury monitored by optical imaging. Int J Mol Med 2014, 33, 1055-1063, doi:10.3892/ijmm.2014.1663.

42. Gazdic, M.; Volarevic, V.; Arsenijevic, N.; Stojkovic, M. Mesenchymal Stem Cells: A Friend or Foe in Immune-Mediated Diseases. Stem Cell Reviews and Reports 2015, 11, 280-287, doi:10.1007/s12015-014-9583-3.

43. Otero-Ortega, L.; Gómez de Frutos, M.C.; Laso-García, F.; Rodríguez-Frutos, B.; Medina-Gutiérrez, E.; López, J.A.; Vázquez, J.; Díez-Tejedor, E.; Gutiérrez-Fernández, M. Exosomes promote restoration after an experimental animal model of intracerebral hemorrhage. $J$ Cereb Blood Flow Metab 2017, 38, 767-779, doi:10.1177/0271678X17708917.

44. Alvarez-Erviti, L.; Seow, Y.; Yin, H.; Betts, C.; Lakhal, S.; Wood, M.J.A. Delivery of siRNA to the mouse brain by systemic injection of targeted exosomes. Nature Biotechnology 2011, 29, 341-345, doi:10.1038/nbt.1807.

45. Kooijmans, S.A.A.; Aleza, C.G.; Roffler, S.R.; van Solinge, W.W.; Vader, P.; Schiffelers, R.M. Display of GPI-anchored anti-EGFR nanobodies on extracellular vesicles promotes tumour cell targeting. null 2016, 5, 31053, doi:10.3402/jev.v5.31053.

46. Chen, T.S.; Lai, R.C.; Lee, M.M.; Choo, A.B.H.; Lee, C.N.; Lim, S.K. Mesenchymal stem cell secretes microparticles enriched in pre-microRNAs. Nucleic Acids Res 2010, 38, 215-224, doi:10.1093/nar/gkp857.

47. Valadi, H.; Ekström, K.; Bossios, A.; Sjöstrand, M.; Lee, J.J.; Lötvall, J.O. Exosomemediated transfer of mRNAs and microRNAs is a novel mechanism of genetic exchange between cells. Nature Cell Biology 2007, 9, 654.

48. Reis, L.A.; Borges, F.T.; Simões, M.J.; Borges, A.A.; Sinigaglia-Coimbra, R.; Schor, N. Bone marrow-derived mesenchymal stem cells repaired but did not prevent gentamicin-induced acute kidney injury through paracrine effects in rats. PLoS One 2012, 7, e44092-e44092, doi:10.1371/journal.pone.0044092.

49. Phinney, D.G.; Di Giuseppe, M.; Njah, J.; Sala, E.; Shiva, S.; St Croix, C.M.; Stolz, D.B.; Watkins, S.C.; Di, Y.P.; Leikauf, G.D.; et al. Mesenchymal stem cells use extracellular vesicles to outsource mitophagy and shuttle microRNAs. Nat Commun 2015, 6, 8472-8472, doi:10.1038/ncomms9472.

50. Nakamura, Y.; Miyaki, S.; Ishitobi, H.; Matsuyama, S.; Nakasa, T.; Kamei, N.; Akimoto, T.; Higashi, Y.; Ochi, M. Mesenchymal-stem-cell-derived exosomes 
accelerate skeletal muscle regeneration. FEBS Letters 2015, 589, 1257-1265, doi:10.1016/j.febslet.2015.03.031.

51. Zhou, Y.; Xu, H.; Xu, W.; Wang, B.; Wu, H.; Tao, Y.; Zhang, B.; Wang, M.; Mao, F.; Yan, Y.; et al. Exosomes released by human umbilical cord mesenchymal stem cells protect against cisplatin-induced renal oxidative stress and apoptosis in vivo and in vitro. Stem Cell Res Ther 2013, 4, 34-34, doi:10.1186/scrt194.

52. Zhou, Y.; Yamamoto, Y.; Itaya, T.; Xiao, Z.-D.; Ochiya, T. Distinct Mirna Expression Patterns of Extracellular Vesicles Derived From 4 Types of Mesenchymal Stem Cells. J Stem Cell Res Ther 2018, 8, 8, doi:10.4172/2157-7633.1000415.

53. Liu, Y.; Guan, H.; Zhang, J.-L.; Zheng, Z.; Wang, H.-T.; Tao, K.; Han, S.-C.; Su, L.L.; Hu, D. Acute downregulation of miR-199a attenuates sepsis-induced acute lung injury by targeting SIRT1. American Journal of Physiology-Cell Physiology 2018, 314, C449-C455, doi:10.1152/ajpcell.00173.2017.

54. Chen, Z.; Dong, W.-H.; Chen, Q.; Li, Q.-G.; Qiu, Z.-M. Downregulation of miR-199a$3 p$ mediated by the CtBP2-HDAC1-FOXP3 transcriptional complex contributes to acute lung injury by targeting NLRP1. Int J Biol Sci 2019, 15, 2627-2640, doi:10.7150/ijbs.37133.

55. Ranganathan, P.; Ngankeu, A.; Zitzer, N.C.; Leoncini, P.; Yu, X.; Casadei, L.; Challagundla, K.; Reichenbach, D.K.; Garman, S.; Ruppert, A.S.; et al. Serum miR-29a Is Upregulated in Acute Graft-versus-Host Disease and Activates Dendritic Cells through TLR Binding. J Immunol 2017, 198, 2500-2512, doi:10.4049/jimmunol.1601778.

56. Yang, Y.-L.; Kuo, H.-C.; Wang, F.-S.; Huang, Y.-H. MicroRNA-29a Disrupts DNMT3b to Ameliorate Diet-Induced Non-Alcoholic Steatohepatitis in Mice. Int J Mol Sci 2019, 20, 1499, doi:10.3390/ijms20061499.

57. Ge, Q.-M.; Huang, C.-M.; Zhu, X.-Y.; Bian, F.; Pan, S.-M. Differentially expressed miRNAs in sepsis-induced acute kidney injury target oxidative stress and mitochondrial dysfunction pathways. PLoS One 2017, 12, e0173292-e0173292, doi:10.1371/journal.pone.0173292.

58. Li, H.; Shi, H.; Gao, M.; Ma, N.; Sun, R. Long non-coding RNA CASC2 improved acute lung injury by regulating miR-144-3p/AQP1 axis to reduce lung epithelial cell apoptosis. Cell Biosci 2018, 8, 15-15, doi:10.1186/s13578-018-0205-7.

59. Bahudhanapati, H.; Tan, J.; Dutta, J.A.; Strock, S.B.; Sembrat, J.; Àlvarez, D.; Rojas, M.; Jäger, B.; Prasse, A.; Zhang, Y.; et al. MicroRNA-144-3p targets relaxin/insulinlike family peptide receptor 1 (RXFP1) expression in lung fibroblasts from patients with idiopathic pulmonary fibrosis. J Biol Chem 2019, 294, 5008-5022, doi:10.1074/jbc.RA118.004910.

60. D’souza, N.; Burns, J.S.; Grisendi, G.; Candini, O.; Veronesi, E.; Piccinno, S.; Horwitz, E.M.; Paolucci, P.; Conte, P.; Dominici, M. MSC and Tumors: Homing, Differentiation, and Secretion Influence Therapeutic Potential. In Mesenchymal Stem Cells - Basics and Clinical Application II; Weyand, B., Dominici, M., Hass, R., Jacobs, R., Kasper, C., Eds.; Springer Berlin Heidelberg: Berlin, Heidelberg, 2013; pp. 209-266 ISBN 9783-642-37944-4.

61. Maeda, Y.; Davé, V.; Whitsett, J.A. Transcriptional Control of Lung Morphogenesis. Physiological Reviews 2007, 87, 219-244, doi:10.1152/physrev.00028.2006.

62. Vranken, B.E.V.; Romanska, H.M.; Polak, J.M.; Rippon, H.J.; Shannon, J.M.; Bishop, A.E. Coculture of Embryonic Stem Cells with Pulmonary Mesenchyme: A Microenvironment That Promotes Differentiation of Pulmonary Epithelium. Tissue Engineering 2005, 11, 1177-1187, doi:10.1089/ten.2005.11.1177.

63. Ware, L.B.; Matthay, M.A. Keratinocyte and hepatocyte growth factors in the lung: roles in lung development, inflammation, and repair. American Journal of Physiology- 
Lung Cellular and Molecular Physiology 2002, 282, L924-L940, doi:10.1152/ajplung.00439.2001.

64. Moore, B.B.; Peters-Golden, M.; Christensen, P.J.; Lama, V.; Kuziel, W.A.; Paine, R.; Toews, G.B. Alveolar epithelial cell inhibition of fibroblast proliferation is regulated by MCP-1/CCR2 and mediated by PGE2. American Journal of Physiology-Lung Cellular and Molecular Physiology 2003, 284, L342-L349, doi:10.1152/ajplung.00168.2002.

65. Ray, P. Protection of epithelial cells by keratinocyte growth factor signaling. Proc Am Thorac Soc 2005, 2, 221-225, doi:10.1513/pats.200502-012AC.

66. Badri, L.; Walker, N.M.; Ohtsuka, T.; Wang, Z.; Delmar, M.; Flint, A.; Peters-Golden, M.; Toews, G.B.; Pinsky, D.J.; Krebsbach, P.H.; et al. Epithelial interactions and local engraftment of lung-resident mesenchymal stem cells. Am J Respir Cell Mol Biol 2011, 45, 809-816, doi:10.1165/rcmb.2010-0446OC.

67. Salazar, K.D.; Lankford, S.M.; Brody, A.R. Mesenchymal stem cells produce Wnt isoforms and TGF-beta1 that mediate proliferation and procollagen expression by lung fibroblasts. Am J Physiol Lung Cell Mol Physiol 2009, 297, L1002-L1011, doi:10.1152/ajplung.90347.2008.

68. Zhu, Y.-G.; Feng, X.-M.; Abbott, J.; Fang, X.-H.; Hao, Q.; Monsel, A.; Qu, J.-M.; Matthay, M.A.; Lee, J.W. Human mesenchymal stem cell microvesicles for treatment of Escherichia coli endotoxin-induced acute lung injury in mice. Stem Cells 2014, 32, 116-125, doi:10.1002/stem.1504.

69. Gennai, S.; Monsel, A.; Hao, Q.; Park, J.; Matthay, M.A.; Lee, J.W. Microvesicles Derived From Human Mesenchymal Stem Cells Restore Alveolar Fluid Clearance in Human Lungs Rejected for Transplantation. Am J Transplant 2015, 15, 2404-2412, doi:10.1111/ajt.13271.

70. Li, J. wei; Wei, L.; Han, Z.; Chen, Z. Mesenchymal stromal cells-derived exosomes alleviate ischemia/reperfusion injury in mouse lung by transporting anti-apoptotic miR21-5p. European Journal of Pharmacology 2019, 852, 68-76, doi:10.1016/j.ejphar.2019.01.022.

71. Bari, E.; Ferrarotti, I.; Di Silvestre, D.; Grisoli, P.; Barzon, V.; Balderacchi, A.; Torre, M.L.; Rossi, R.; Mauri, P.; Corsico, A.G.; et al. Adipose Mesenchymal Extracellular Vesicles as Alpha-1-Antitrypsin Physiological Delivery Systems for Lung Regeneration. Cells 2019, 8, 965, doi:10.3390/cells8090965.

72. Hao, Q.; Gudapati, V.; Monsel, A.; Park, J.H.; Hu, S.; Kato, H.; Lee, J.H.; Zhou, L.; He, H.; Lee, J.W. Mesenchymal Stem Cell-Derived Extracellular Vesicles Decrease Lung Injury in Mice. J. Immunol. 2019, 203, 1961, doi:10.4049/jimmunol.1801534.

73. Grabiec, A.M.; Hussell, T. The role of airway macrophages in apoptotic cell clearance following acute and chronic lung inflammation. Seminars in Immunopathology 2016, 38, 409-423, doi:10.1007/s00281-016-0555-3.

74. Stone, M.L.; Zhao, Y.; Robert Smith, J.; Weiss, M.L.; Kron, I.L.; Laubach, V.E.; Sharma, A.K. Mesenchymal stromal cell-derived extracellular vesicles attenuate lung ischemia-reperfusion injury and enhance reconditioning of donor lungs after circulatory death. Respir Res 2017, 18, 212-212, doi:10.1186/s12931-017-0704-9.

75. Cho, K.-S.; Kang, S.A.; Kim, S.-D.; Mun, S.-J.; Yu, H.S.; Roh, H.-J. Dendritic cells and M2 macrophage play an important role in suppression of Th2-mediated inflammation by adipose stem cells-derived extracellular vesicles. Stem Cell Research 2019, 39, 101500, doi:10.1016/j.scr.2019.101500.

76. Zheng, G.; Huang, L.; Tong, H.; Shu, Q.; Hu, Y.; Ge, M.; Deng, K.; Zhang, L.; Zou, B.; Cheng, B.; et al. Treatment of acute respiratory distress syndrome with allogeneic adipose-derived mesenchymal stem cells: a randomized, placebo-controlled pilot study. Respir Res 2014, 15, 39-39, doi:10.1186/1465-9921-15-39. 
77. Galstian, G.M.; Parovichnikova, E.N.; Makarova, P.M.; Kuzmina, L.A.; Troitskaya, V.V.; Gemdzhian, E.; Drize, N.I.; Savchenko, V.G. The Results of the Russian Clinical Trial of Mesenchymal Stromal Cells (MSCs) in Severe Neutropenic Patients (pts) with Septic Shock (SS) (RUMCESS trial). Blood 2015, 126, 2220-2220, doi:10.1182/blood.V126.23.2220.2220.

78. Wilson, J.G.; Liu, K.D.; Zhuo, H.; Caballero, L.; McMillan, M.; Fang, X.; Cosgrove, K.; Vojnik, R.; Calfee, C.S.; Lee, J.-W.; et al. Mesenchymal stem (stromal) cells for treatment of ARDS: a phase 1 clinical trial. Lancet Respir Med 2015, 3, 24-32, doi:10.1016/S2213-2600(14)70291-7.

79. Simonson, O.E.; Mougiakakos, D.; Heldring, N.; Bassi, G.; Johansson, H.J.; Dalén, M.; Jitschin, R.; Rodin, S.; Corbascio, M.; El Andaloussi, S.; et al. In Vivo Effects of Mesenchymal Stromal Cells in Two Patients With Severe Acute Respiratory Distress Syndrome. Stem Cells Transl Med 2015, 4, 1199-1213, doi:10.5966/sctm.2015-0021.

80. Matthay, M.A.; Calfee, C.S.; Zhuo, H.; Thompson, B.T.; Wilson, J.G.; Levitt, J.E.; Rogers, A.J.; Gotts, J.E.; Wiener-Kronish, J.P.; Bajwa, E.K.; et al. Treatment with allogeneic mesenchymal stromal cells for moderate to severe acute respiratory distress syndrome (START study): a randomised phase 2a safety trial. Lancet Respir Med $\mathbf{2 0 1 9}$, 7, 154-162, doi:10.1016/S2213-2600(18)30418-1.

81. Zhang, H.; Li, Y.; Slutsky, A.S. Precision medicine for cell therapy in acute respiratory distress syndrome. The Lancet Respiratory Medicine 2019, 7, e13, doi:10.1016/S22132600(19)30089-X.

82. Sengupta, V.; Sengupta, S.; Lazo, A.; Woods, P.; Nolan, A.; Bremer, N. Exosomes Derived from Bone Marrow Mesenchymal Stem Cells as Treatment for Severe COVID19. Stem Cells Dev 2020, 29, 747-754, doi:10.1089/scd.2020.0080.

83. Derwall, M.; Martin, L.; Rossaint, R. The acute respiratory distress syndrome: pathophysiology, current clinical practice, and emerging therapies. null 2018, 12, 1021 1029, doi:10.1080/17476348.2018.1548280.

84. Yi, X.; Wei, X.; Lv, H.; An, Y.; Li, L.; Lu, P.; Yang, Y.; Zhang, Q.; Yi, H.; Chen, G. Exosomes derived from microRNA-30b-3p-overexpressing mesenchymal stem cells protect against lipopolysaccharide-induced acute lung injury by inhibiting SAA3. Experimental Cell Research 2019, 383, 111454, doi:10.1016/j.yexcr.2019.05.035.

85. Hare, J.M.; Fishman, J.E.; Gerstenblith, G.; DiFede Velazquez, D.L.; Zambrano, J.P.; Suncion, V.Y.; Tracy, M.; Ghersin, E.; Johnston, P.V.; Brinker, J.A.; et al. Comparison of allogeneic vs autologous bone marrow-derived mesenchymal stem cells delivered by transendocardial injection in patients with ischemic cardiomyopathy: the POSEIDON randomized trial. JAMA 2012, 308, 2369-2379, doi:10.1001/jama.2012.25321.

86. Cheng, R.-J.; Xiong, A.-J.; Li, Y.-H.; Pan, S.-Y.; Zhang, Q.-P.; Zhao, Y.; Liu, Y.; Marion, T.N. Mesenchymal Stem Cells: Allogeneic MSC May Be Immunosuppressive but Autologous MSC Are Dysfunctional in Lupus Patients. Front Cell Dev Biol 2019, 7, 285-285, doi:10.3389/fcell.2019.00285.

87. Ciuffreda, M.C.; Malpasso, G.; Musarò, P.; Turco, V.; Gnecchi, M. Protocols for in vitro Differentiation of Human Mesenchymal Stem Cells into Osteogenic, Chondrogenic and Adipogenic Lineages. In Mesenchymal Stem Cells: Methods and Protocols; Gnecchi, M., Ed.; Springer New York: New York, NY, 2016; pp. 149-158 ISBN 978-1-4939-3584-0.

88. Heo, J.S.; Choi, Y.; Kim, H.-S.; Kim, H.O. Comparison of molecular profiles of human mesenchymal stem cells derived from bone marrow, umbilical cord blood, placenta and adipose tissue. Int J Mol Med 2016, 37, 115-125, doi:10.3892/ijmm.2015.2413.

89. Haraszti, R.A.; Miller, R.; Stoppato, M.; Sere, Y.Y.; Coles, A.; Didiot, M.-C.; Wollacott, R.; Sapp, E.; Dubuke, M.L.; Li, X.; et al. Exosomes Produced from 3D 
Cultures of MSCs by Tangential Flow Filtration Show Higher Yield and Improved Activity. Mol Ther 2018, 26, 2838-2847, doi:10.1016/j.ymthe.2018.09.015.

90. Cao, J.; Wang, B.; Tang, T.; Lv, L.; Ding, Z.; Li, Z.; Hu, R.; Wei, Q.; Shen, A.; Fu, Y.; et al. Three-dimensional culture of MSCs produces exosomes with improved yield and enhanced therapeutic efficacy for cisplatin-induced acute kidney injury. Stem Cell Res Ther 2020, 11, 206-206, doi:10.1186/s13287-020-01719-2.

91. Dinh, P.-U.C.; Paudel, D.; Brochu, H.; Popowski, K.D.; Gracieux, M.C.; Cores, J.; Huang, K.; Hensley, M.T.; Harrell, E.; Vandergriff, A.C.; et al. Inhalation of lung spheroid cell secretome and exosomes promotes lung repair in pulmonary fibrosis. Nat Commun 2020, 11, 1064-1064, doi:10.1038/s41467-020-14344-7.

92. Chen, Y.-S.; Lin, E.-Y.; Chiou, T.-W.; Harn, H.-J. Exosomes in clinical trial and their production in compliance with good manufacturing practice. Ci Ji Yi Xue Za Zhi 2019, 32, 113-120, doi:10.4103/tcmj.tcmj_182_19. 